\title{
Robust Bi-level Model for Optimal Allocation and Design of Power System Stabilizer in MultiMachine Power Systems
}

\author{
Farhad Samadi Gazijahani ${ }^{1}$, Ata Ajoul Abadi ${ }^{2}$ and Amin Safari ${ }^{3}$ \\ ${ }^{1,2,3}$ Department of Electrical Engineering, Azarbaijan Shahid Madani University, \\ Tabriz, Iran.
}

\begin{abstract}
This paper presents a new optimized and systematic bi-level approach for the optimal robust allocation and design of Power System Stabilizers (PSS) in order to enhance system stability and damping of low frequency oscillation (LFO) modes in the multimachine systems. The proposed approach provides full ranked optimal conditions for the PSS allocation which provide complete controllability of the system. The proposed technique is based on the use of controllability gramian and participation factor. Moreover, this study proposes a new linear power system model for optimal PSSs design which can consider both local and inter-area modes of the system that the proposed linear model is called Expanded Single-Machine Infinite-Bus (ESMIB) system. The tuning problem of stabilizer parameters is formulated as an optimization problem which is solved by Cuckoo Optimization Algorithm (COA). The eigenvalues based objective function involving damping ratio and damping factor of LFO modes are considered for the PSSs design problem. The stabilization performance of the designed PSSs has been verified in two case study standard models (3 and 10 Machines). The simulation results show that the proposed model can be considered as an efficient tool in optimal allocation and design of PSS in the multi machine environment.
\end{abstract}

Keywords: Power System Stabilizer, Expanded Single-Machine Infinite-Bus, Optimal Allocation, Controllability Gramian, COA

\section{Introduction}

Power systems are complex non-linear and often exhibit low-frequency electromechanical oscillations due to insufficient damping caused by adverse operating conditions. Low frequency oscillations (LFOs) refer to the angle oscillations of the generator rotor after a disturbance. The oscillations that occur between a single generator or a single power plant and the rest of the power system are called local oscillations which oscillate in a frequency range of $0.8-2 \mathrm{~Hz}$. Inter-area oscillations, ranging $0.2-0.8 \mathrm{~Hz}$, appear from two or more groups of generators in different areas, swinging against each other. Usually, these oscillations occur in the power systems connected via weak power transmission lines. In power systems under stress, damping of LFO is generally low. Therefore, supplementary stabilizers are used to damp these oscillations. In most power systems, local oscillations are often damped well due to installing local PSS while interarea oscillations are often weakly damped, because the input control signals used in these PSSs are local signals and do not often have good controllability over some of the critical inter-area modes. Small amplitude power oscillations are having low frequency often persisted for long periods of time. In some cases, this leads to a limitation on the amount of the power to be transmitted via transmission lines. The PSSs are auxiliary control devices installed on synchronous generators and provide control signals to enhance the system damping and extend power transfer via transmission lines [1,2]. With the increase

Received (May 23, 2017), Review Result (August 18, 2017), Accepted (August 22, 2017) 
of the scale and complexity of the interconnected power systems, the problems on the various potential power swings, which have the important impact on the system stability and security operation [3-5].

The first step in designing such a controller is to find its optimum location. Though, there is a common perception that the application of PSS is almost mandatory required on all generators in the modern power network. Conventional power system stabilizer, as a lead-lag compensator with two categories of single input and dual inputs, is mainly designed based on using a linear model and considering one operating point. In general, the PSS problem can be divided into two major categories: 1) optimal allocation and 2) optimal design. Several methods to determine the best location for PSS installation have been studied [6-9] including sequential methods such as outlined in [10] taking into account the presence of PSSs that were already installed. The sequential placement does not always determine the best multiple PSS placements because the placement order can affect the results. Other methods require eigenvalue calculations after PSS installation at each generator. Also, the optimal design of PSSs is frequently adjusted by optimization algorithms such as PSO [11], genetic algorithm [12], simulated annealing [13], chaotic algorithm [14], bacteria foraging [15] and other tuning algorithms such as $H_{\infty}$ [16], sequential linear programming [17] and LMI technique [18]. In the second group, neural networks [19], wavelet based neural network controllers [20], neuro-fuzzy controller [21] and fuzzy logic controllers [22] are used as adaptive power system stabilizers. However, allocation and design of PSS are reported in previous literature, but coordinately optimal allocation and design of PSS is not reported in current literature. To fill-out this gap, this paper proposes an integrated bi-level method for optimal placement and design of PSS that provides full controllability of the system. The proposed technique is based on the use of controllability gramian and participation factor to characterize large power system and extended to optimal placement of PSS in power systems. The main idea is to select the controllers corresponding to the minimum energy needed to control the most significant modes of the system. Moreover, a new model based on a linear model of the dominant generator has been proposed for designing of the PSS in which both local and inter-area mode oscillations have been considered. The proposed linear model is called ExpandedSingle-Machine-Infinite-Bus (ESMIB) system. As a result, this paper has been shown that the damping performance of the designed PSS based on the proposed model has been improved in comparison with the designed PSS based on the SMIB model. In the application of the proposed method, the paper utilized the IEEE 9-bus test system [23] for investigating the proposed approach in details and New England test System for validating the proposed approach [24].

The major contributions of this paper are summarized as below:

- Considering simultaneously PSS allocation and tuning in the multi-machine system.

- Proposing a new robust bi-level model for PSS planning problem.

- Developing a new linear model relies on ESMIB system to consider both local and inter-area oscillation modes.

- Applying integrated full ranked optimal conditions for the PSS planning problem based on controllability gramian and participation factor.

- Formulation the proposed model as Mixed Integer Non-Linear Programming (MINLP) and performing a new optimization algorithm namely cuckoo optimization algorithm in order to adjust the PSS parameters.

- Implementation the proposed model on the two diverse multi-machine systems to demonstrate the effectiveness and applicability of the presented approach.

The paper is organized as follows: Section 2 explains the theory and PSS allocation concepts; design of PSS is explained in Section 3; discusses the numerical results in Section 4 and Section 5 shows the conclusions. 


\section{Theory and Allocation of PSS}

The theoretical formulation of the proposed architecture for PSS allocation is illustrated in this section. The methodology consists of system analysis based on combined small signal and transient stability property of the power system. First, the small signal stability based architecture is demonstrated.

\subsection{Problem Formulation and Theoretical Analysis}

Consider a system described in state-space form by: $\dot{x}(t)=A \cdot x(t)+B \cdot u(t)$

$$
y(t)=C \cdot x(t)
$$

We assume that equation (1) is controllable and observable. The transient controllability function of a continuous-time linear system is defined as:

$$
L c(X, T)=\min _{u, x(0)}=x_{2} \frac{1}{2} \int_{-T}^{0}\|u(\tau)\|^{2} d \tau, x(-T)=0
$$

The transient controllability function is given by:

$$
\begin{gathered}
L c(X, T)=\frac{1}{2} X^{T} W c^{-1}(T) X \\
W c(T)=\int_{-T}^{0} e^{A t} B B^{T} e^{A^{T} t} d t \\
x(t)=\int_{0}^{\tau} e^{A(t-\tau)} B u(\tau) d \tau
\end{gathered}
$$

A necessary and sufficient condition of controllability of the system on the interval $[0$, $\tau]$ for any $T>0$ is ranked $[B, A B, A n-1 B]=n$. Controllability is characterized by the following conditions, $\eta \in \Re n$. Since the controllability means the set of all attainable vectors $x \in \mathfrak{R} n$ corresponding to all possible choices of control $u$ is the whole space $\mathfrak{R} n$ and the only vector orthogonal to the whole space is the zero vector. The following matrix is called the controllability gramian for the system.

$$
W c(T)=\int_{0}^{\tau} e^{A(t-\tau)} B B^{T} u(\tau) d \tau
$$

Considering system (1) is fully controllable on $[0, T]$ if and only if rank $W c=n$, then the quadratic form can be written as:

$$
\begin{gathered}
\eta^{T} W_{C \eta}=\int_{0}^{\tau} \eta^{T} e^{A(t-\tau)} B B^{T} e^{A(t-\tau)} \eta d \tau=\int_{0}^{\tau}\|u(\tau)\|^{2} d \tau \geq 0 \\
u(\tau)=B^{T} e^{A(t-\tau)} \eta \\
\eta^{T} W_{C \eta}=0
\end{gathered}
$$

Also, for controlling the system, controllability matrix $W_{C}(t)$ is positive definite solution, obtained at time $t=T$, of the following differential Lyapunov equations [20].

$$
-W_{C}(t)+A W_{C}(t)+W_{C}(t) A^{T}=-B B^{T} W_{C}(0)=0
$$

If the system in equation (1) is asymptotically stable around the origin, $L_{c}$ is finite. When $\rightarrow \infty: \lim _{T \rightarrow \infty} W_{C}(T)=\overline{W_{C}}, \bar{W}_{C}$ is obtained as the unique positive definite solution of the following Lyapunov equation.

$$
A W_{C}^{2}+W_{C}^{2} A^{T}+B B^{T}=0
$$

Since the controllability matrix $W c$ depends on the control input matrix $\mathrm{B}$, the control energy can be affected by properly choosing this control input matrix. From equations (1) to $(11)$, it is suggested that in order to minimize the control input energy $u(\tau)$ is proportional to $\left(W_{C}^{-1}\right)$, therefore, we have to minimize $\left(W_{C}^{-1}\right)$ or equivalently to maximize $W_{C}$ in the sense of given matrix norm. The most appropriate generator for PSS placement is the one that provides maximum energy which corresponds to the trace of $W c$. The energy index can be represented as:

$$
E_{i}=\operatorname{trace}\left\{\left(W_{C}\right)\right\}
$$

The index $E i$ can be calculated for each generator and the generator corresponding to the maximum value of the index is the most suitable one for placing the PSS. Further from equation (12) generator participation can be developed for that current scenario as

$$
P I_{i}=E_{i} / \max \left(E_{i}\right)
$$




\subsection{Model Reduction for Larger Power System}

For larger power system, a reduced model has to be developed so that we can analyze the optimal placement scenarios. Consider a linear system given by equation (1), which is assumed to be asymptotically stable, both stable and detectable. It can be said that there exists a regular transformation matrix $P$ such that:

$$
\begin{gathered}
\bar{x}=P A P^{-1} \bar{x}+P S B u=\bar{A} \bar{x}+\bar{B} u^{\bar{x}=P . x} \\
y=C P^{-1} \bar{x}=\bar{C} \bar{x}
\end{gathered}
$$

Matrix $P$ can be obtained by performing Cholesky decomposition of controllability gramian matrix $\left(W_{C}\right)$. Then, balance system gramian can be obtained as follows:

$$
W_{C}=L_{C} W_{C} L_{C}^{T}
$$

Based on equation(16) computation of singular value decomposition for factorizing as follows:

$$
L_{C} W_{O} L_{C}^{T}, i . e L_{C} W_{O} L_{C}^{T}=U^{T} \Sigma^{2} U
$$

That is a unitary matrix (i.e., $\left.U U^{T}=U^{T} U=I\right)$ and $\sum=\operatorname{diag}\left(\sigma_{1}, \sigma_{2}, \ldots, \sigma_{n}\right)$ and $\sigma_{\bar{i}}$ are the Hankel singular values (HSV), i.e. the square roots of the eigenvalues $\lambda_{i}\left(W_{r} W_{o}\right)$.

$$
T=L_{C}^{T} U \Sigma^{-1 / 2}
$$

Forming balanced realization $S_{b}$ of the system transformation can be found as follows:

$$
\begin{gathered}
S_{b}=\left\{T^{-1} A T, T^{-1} B, C T\right\} \\
S_{k}=\left\{A_{k} B_{k}, C_{k}\right\}=\left\{T_{l} A T_{r}, T_{l} B, C T_{r}\right\}
\end{gathered}
$$

Where, $T_{I}$ is comprised of first $k$ row of $T$ and $T_{r}$ is comprised of first $k$ columns of $T$. The error between original system $S$ and the reduced order system $S_{k}$ satisfies the following equations:

$$
\begin{gathered}
\left\|G(s)-G_{k}(s)\right\|_{\infty} \leq 2 \sum_{i=k+1}^{n} \sigma_{i} \\
\left\|G(S)-G_{k}(S)\right\|_{\infty} \geq \sigma_{k}
\end{gathered}
$$

The reduced order system can be presented as:

$$
X_{\text {red }}^{*}=A_{\text {red }} X_{r e d}+B_{\text {red }} u \quad y=C_{r e d} X_{r e d}+D u
$$

\subsection{Procedure for Optimal Placement of Power System Stabilizer}

The procedure for optimal PSS allocation as well as participation of generators can be designed as follows.

- Step1: First develop a linearized model of the power grid based on (14) to (23).

- Step2: Calculate the controllability gramian $W c$ without controller from (3) for that specific scenario and time duration. Then, calculate the $E_{\mathrm{i}}$ and $P I_{\mathrm{i}}$ for all generators from (12). Select the generator with maximum $E i$ for placement of PSS with nominal values.

- Step3: Tune the PSS based on the proposed methodology (discussed in section 3) for the generator where the PSS is placed.

- Step4: From energy participation index $P I_{i}$, updates the controller coefficient matrix $B$ using (6) for the other generators.

- Step5: Calculate the controllability gramian $W c$ with a controller from (6) and utilizing the control signal $U$ generated based on the selected and tuned PSS.

Overall approach here consists of adding PSSs that will provide additional input control signals with the goal of increasing robustness with respect to disturbances while taking into account the influence of all existing controllers in the whole power system. So that, the problem of choosing optimal placement of controllers to prevent power system from oscillations. This methodology should be continued for each perturbation scenario. From each of the control inputs it can be finalized that the proposed architecture provides improved controllability index and the participation factor. Finally, as the placement of PSS, the index converges to a specific value that provides full controllability. 


\section{PSS Design Based on Proposed New Linear Model}

It would be desirable to create a linear model to analyze the equipments in the power system accurately. For this reason, it is difficult to create a precise model with all details. The depth of the system analysis depends on the size of the power system in question, and the accuracy of the model must be compromised to some degree. Local mode oscillations have been known to occur at the generator connected to the system with tie-lines that are weak in the case of the infinite bus model. However, the inter-area mode occurs in a very large system of interconnected, multi-machine systems, thereby, it is difficult to perform a detailed analysis [25]. The PSSs are very effective controllers in the enhancement of the damping of LFOs, since PSS can increase damping torque for inter-area modes by introducing extra signals into the excitation controllers already provided with generators. The flowchart of the proposed approach for optimal PSS allocation and design is demonstrated in Figure 1. In practical applications, there are different kinds of power plants connected to the network such as renewable power generation and controllable units. Hence, LFOs have different frequencies. For this reason, while PSSs is being designed, it is necessary to consider the effects of the rest of the power system. As a result, it is essential to have a model for designing PSSs as simple as the SMIB system and as exact as the multi-machine power system. The proposed expanded model is shown in Figure 2.

\subsection{Expanded Single Machine Infinite Bus Systems (ESMIB)}

To have a precise and complete linear model of the power system, it is necessary to model all the power system components or the impacts of the rest of the network by one or several inputs. Consider the multi-machine power system, the impacts of different generators on the ith generator are applied by $\mathrm{K}_{1 \mathrm{ij}}$ to $\mathrm{K}_{6 \mathrm{ij}}(\mathrm{i}-\mathrm{j})$, but for example as displayed in Figure 3 all effects and counteracts transmit to the 1rd generator (the dominant generator) that has been connected to the power network. Therefore, the impacts of the rest of the power system can be modeled by measuring variables of the transmission line like the active and reactive powers or the magnitude and angle of the voltage of the determined bus. In the SMIB system, the magnitude and angle of the infinite bus are supposed to be constant. Furthermore, from a practical point of view, the magnitude and angle of bus voltage that has been attached to the 1rd generator via transmission lines oscillate to the effects of power system oscillations.

The infinite bus represents the Thevenin equivalent of a large interconnected power system. The nonlinear equations that describe the generator and excitation system have been represented as follows:

$$
\begin{aligned}
& \hat{\delta}=\omega_{0} \omega \\
& \dot{\omega}=\frac{1}{M}\left(P_{m}-P_{\theta}-D_{m} \omega\right)
\end{aligned}
$$

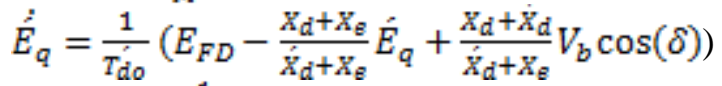

$$
\begin{aligned}
& \hat{E}_{F D}=\frac{1}{T_{A}}\left(K_{A} E_{r e f}-K_{A} V_{t}-E_{F D}\right)
\end{aligned}
$$

Equations (24) to (27) can be linearized for small oscillation around an operating condition as follows.

$$
\begin{gathered}
P_{g i}=\left(\frac{\left(V_{j} \tilde{E}_{q i}\right)}{x_{d i}}\right) \sin \left(\delta_{i}-\theta_{j}\right)+\left(\frac{1}{x_{q i}}-\frac{1}{x_{d i}}\right)\left(\frac{V_{j}^{z}}{2}\right) \sin 2\left(\delta_{i}-\delta \theta_{j}\right) \\
E_{q i}=\left(\frac{x_{d i}}{x_{d i}}\right)\left[\hat{E}_{q i}-V_{j} \cos \left(\delta_{i}-\delta \theta_{j}\right)\right]+V_{j} \cos \left(\delta_{i}-\theta_{j}\right) \\
\bar{V}_{i}=\bar{V}_{j}+j x_{\theta}\left(I_{q i}-j I_{d i}\right) e^{j \delta_{i}} \\
I_{d i}=\left(\hat{E}_{q i}-V_{j} \cos \left(\delta_{i}-\delta \theta_{j}\right)\right) / x_{d i}^{\prime} \mathrm{R}
\end{gathered}
$$




$$
\begin{gathered}
I_{q i}=V_{j} \sin \left(\delta_{i}-\delta \theta_{j}\right) / x_{q i} \\
\Delta P_{g i}=k_{1} \Delta \delta_{i}+k_{2} \Delta E_{q i}+\hat{k}_{1} \Delta \theta_{j}+\hat{k}_{2} \Delta V_{j} \\
\Delta E_{i}=k_{4} \Delta \delta_{i}+\left(1 / k_{3}\right) \Delta \hat{E}_{q i}+\hat{k}_{4} \Delta \theta_{j}+\hat{k}_{3} \Delta V_{j} \\
\Delta V_{i}=k_{5} \Delta \delta_{i}+k_{6} \Delta_{q i}+k_{5} \Delta \theta_{j}+k_{6} \Delta V_{j}
\end{gathered}
$$

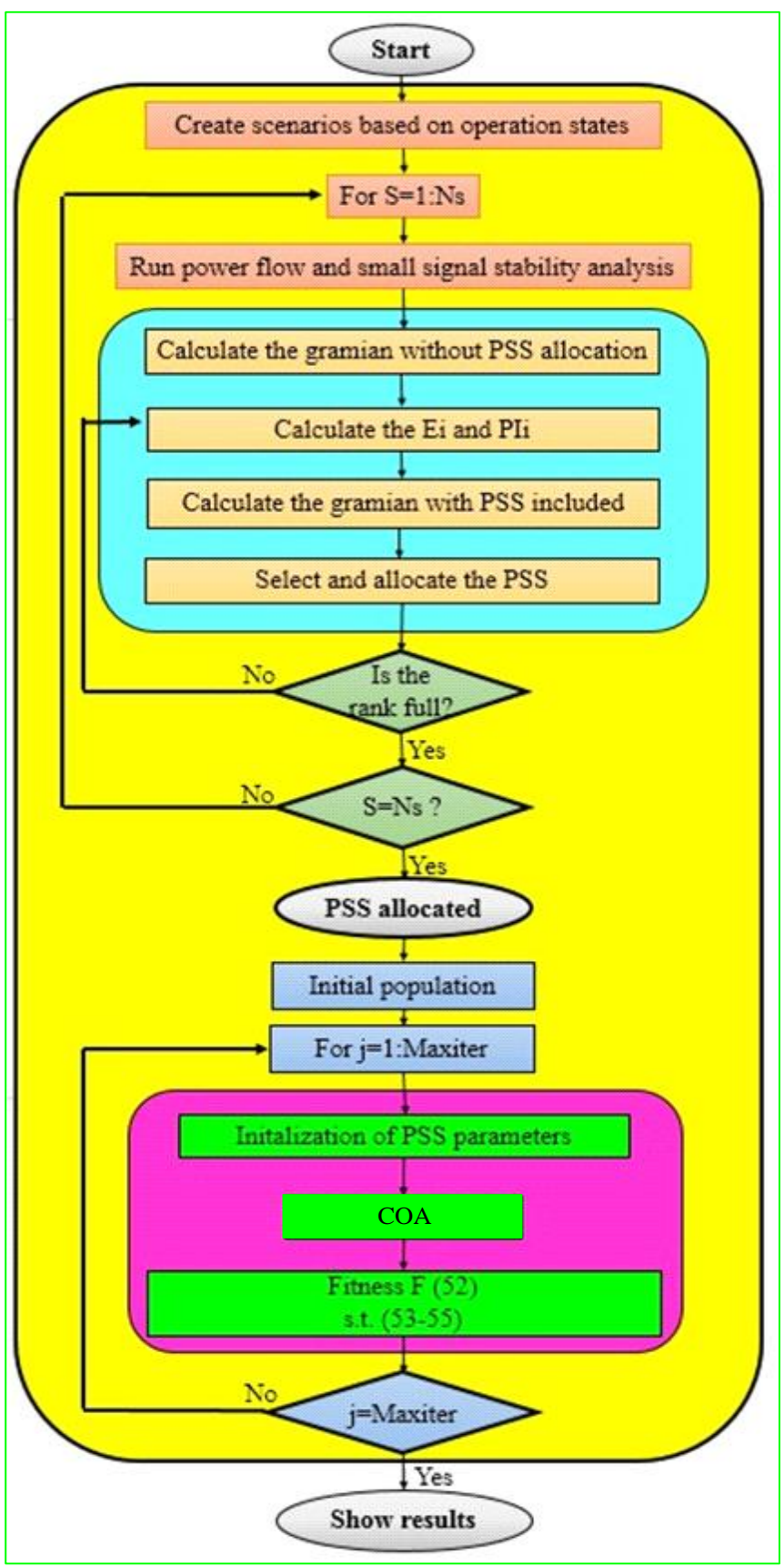

Figure 1. Overall Architecture for PSS Placement and Tuning 


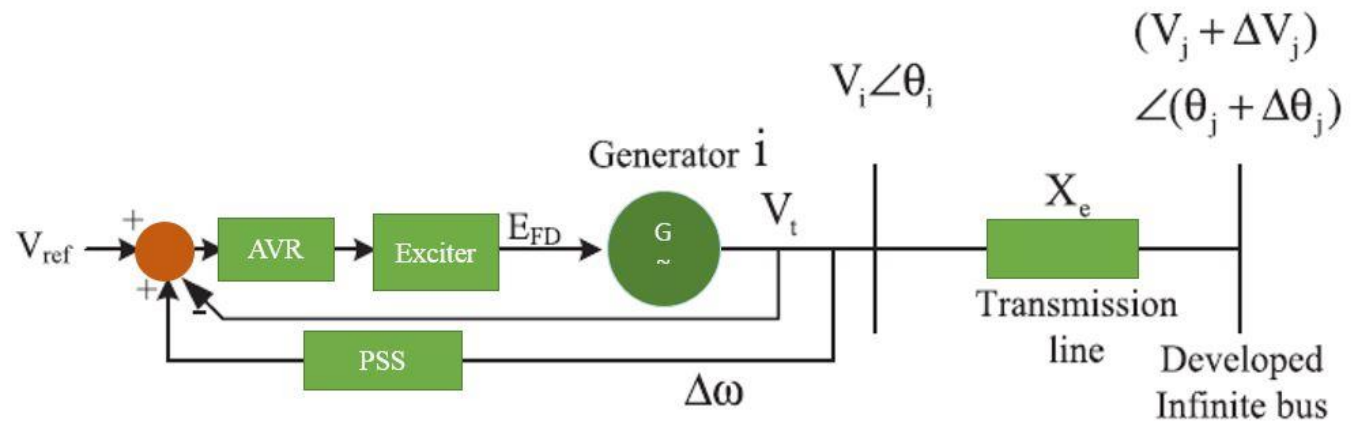

Figure 2. The Expanded-Single-Machine-Normal-Bus System

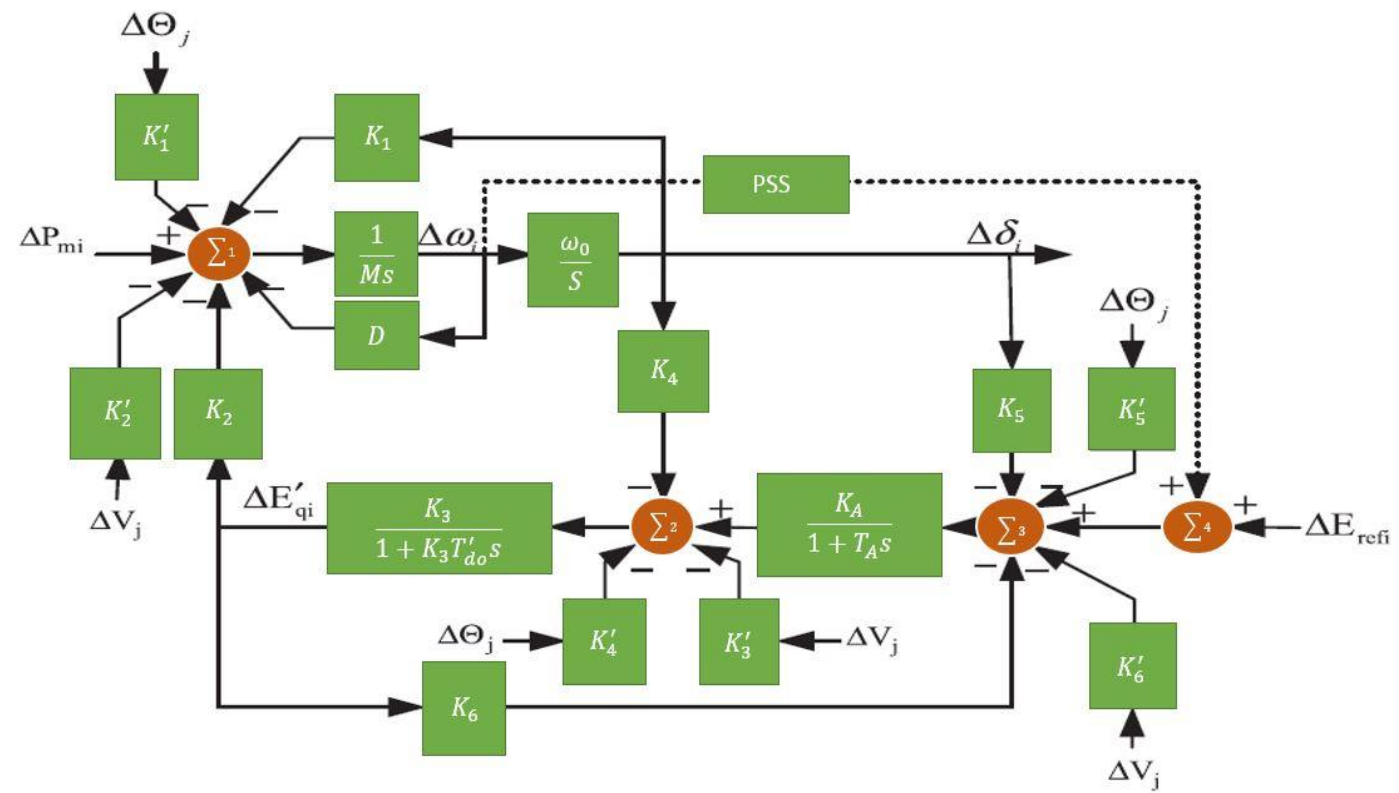

Figure 3. Block Diagram of the ESMIB System

Therefore, if equations (28) to (30) are substituted into equations (24) to (27) and then acquired equations are linearized, block diagram of the ESMIB is obtained (Figure 3). Comparison between Figure 2 and Figure 3 approves that $D_{V j}$ and $D_{h j}$ substituted the rest of the power system which are not modeled in the SMIB system. It means that any kinds of oscillations with different frequencies can be applied to these inputs and then PSSs can be designed regarding the all impacts of the power system. To acquire the constants of the ESMIB system equations (28) to (30) should be linearized around a certain operating point. Therefore, constants $k_{1}, \hat{k}_{1}, k_{2}, \hat{k}_{2}, k_{3}, \hat{k}_{3}, k_{4}, k_{5}, \hat{k}_{5}, k_{6}$ and $\tilde{k}_{6}$ are as follows:

$$
\begin{aligned}
& K_{1}=\left(V_{j}^{*} \hat{E}_{q i}^{s} / x_{d i}^{\prime}\right) \cos \left(\tilde{\delta}_{i}-\delta \ddot{\theta}_{j}\right)+\left(1 / x_{q i}-1 / \dot{x}_{d i}\right) V_{j}^{\prime 2} \cos 2\left(\check{\delta}_{i}-\delta \ddot{\theta}_{j}\right) \\
& K_{2}=\left(V_{1}^{s}=-K_{1} / x_{d i}^{\prime}\right) \sin \left(\check{\delta}_{i}-\delta \check{\theta}_{j}\right) \\
& \tilde{K}_{2}=\left(\tilde{E}_{q i}^{s} / x_{d i}^{\prime}\right) \sin \left(\ddot{\delta}_{i}-\delta \ddot{\theta}_{j}\right)+\left(1 / x_{q i}-1 / \hat{x}_{d i}\right) V_{j}^{2} \sin 2\left(\check{\delta}_{i}-\delta \breve{\theta}_{j}\right) \\
& K_{3}=\overline{x_{d v}} / \overline{x_{d l}} \\
& \tilde{K}_{3}=-\left(\left(x_{d i}-\ddot{x}_{d i}\right) / \dot{x}_{d i}\right) \cos \left(\check{\delta}_{i}-\delta \check{\theta}_{j}\right) \\
& K_{4}=\left(\left(x_{d i}-\ddot{x}_{d i}\right) / \dot{x}_{d i}\right) V_{j}^{=2} \sin \left(\check{\delta}_{i}-\delta \ddot{\theta}_{j}\right) \\
& \stackrel{B}{K}_{4}=-K_{4}
\end{aligned}
$$




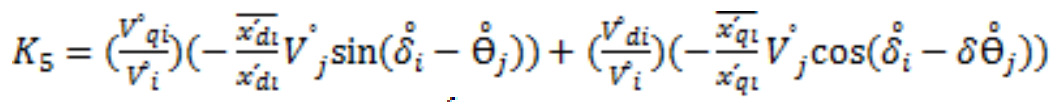

$$
\begin{aligned}
& \dot{K}_{5}=-K_{5} \\
& K_{6}=\left(\frac{V_{q i}^{s i}}{V_{i}^{f}}\left(\frac{x_{\theta}}{x_{d L}^{*}}\right)\right)
\end{aligned}
$$

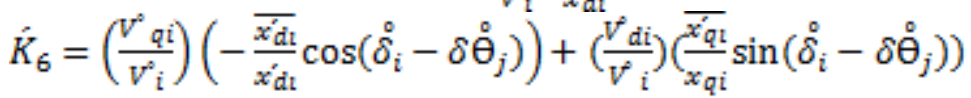

The linearized model of ESMBI system that has been linearized around the certain operating point is given as follows:

$$
\begin{gathered}
\Delta \delta_{i}=\omega_{0} \Delta \omega_{i} \\
\Delta \omega_{i}=-\frac{1}{M}\left(K_{1} \Delta \delta_{i}+\tilde{K}_{1} \Delta \theta_{j}+K_{2} \Delta \tilde{E}_{q i}+\tilde{K}_{2} \Delta V_{j}-\Delta P_{m i}+D_{m i} \Delta \omega_{i}\right) \\
\Delta E_{q i}=-\frac{1}{T_{\text {doi }}}\left(K_{4} \Delta \delta_{i}+\tilde{K}_{4} \Delta \theta_{j}+\frac{1}{K_{\mathrm{g}}} \Delta \hat{E}_{q i}+\hat{K}_{3} \Delta V_{j}-\Delta E_{F D i}\right) \\
\Delta E_{F D i}=-\frac{1}{T_{A}} \Delta E_{F D i}-\frac{K_{A}}{T_{A}}\left(K_{5} \Delta \delta_{i}+\tilde{K}_{5} \Delta \theta_{j}+K_{6} \Delta \tilde{E}_{q i}+\hat{K}_{6} \Delta V_{j}-\Delta E_{r e f i}+U_{p S S}\right.
\end{gathered}
$$

\subsection{Designing of PSS}

In this section, after proposing the ESMIB model for tuning PSSs parameters in the multi-machine power system, it is time to design the parameters of installed PSS of the dominant generator with the ESMIB model. After that, the parameters of the PSS1 are designed by the SMIB model again to analyze and compare the results with the results which have been obtained by the ESMIB model. The block diagram of the PSS1 which is used for G1 is shown in Figure 4.

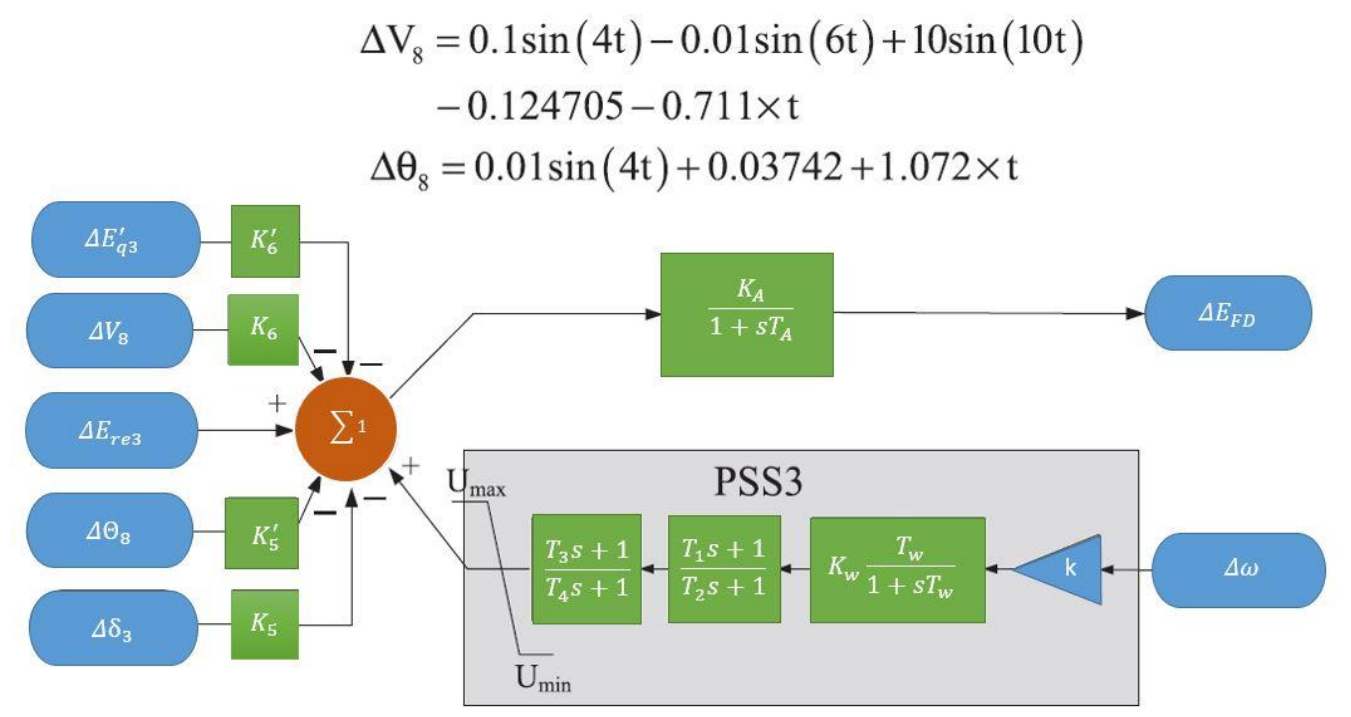

\section{Figure 4. Determination of the Parameters of the PSS1 of the Linear Model of G1 in IEEE 9-Bus Test System}

In this study, Cuckoo optimization algorithm (COA) has been employed to search for the optimal PSS1 parameters. The COA is inspired by the life of a bird family, called Cuckoo [26]. Special lifestyle of these birds and their characteristics in egg laying and breeding has been the basic motivation for development of this new evolutionary optimization algorithm. Similar to other evolutionary methods, the COA starts with an initial population. The cuckoo population, in different societies, is in two types: matures cuckoos and eggs. The effort to survive among cuckoos constitutes the basis of the COA. The survived cuckoo societies immigrate to a better environment and start reproducing and laying eggs. When moving toward the goal point, the cuckoos do not fly all the way 
to the destination habitat. They only fly a part of the way and also have a deviation. This movement and migration of cuckoos are clearly shown in Figure 5. Cuckoos' survival effort hopefully converges to a state that there is only one cuckoo society all with the same profit values.

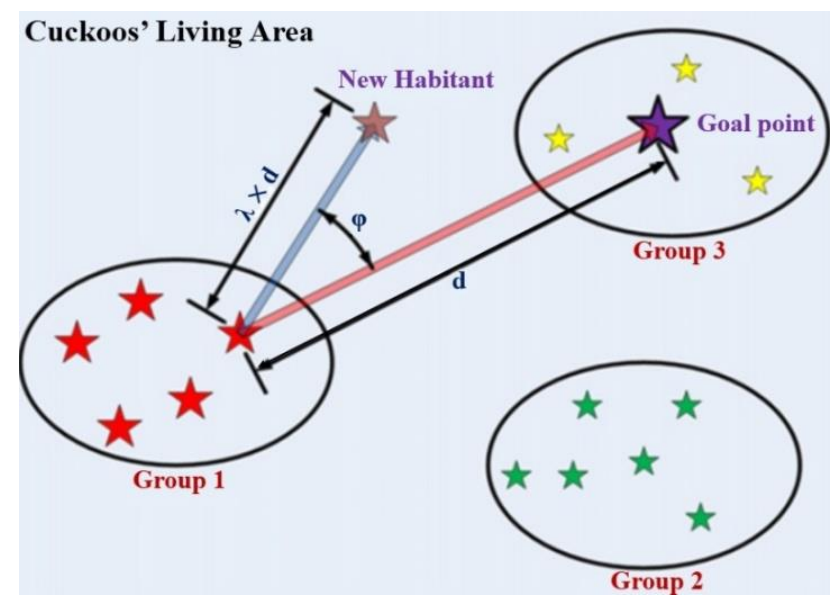

Figure 5. Immigration of a Sample Cuckoo Toward Goal Habitat

The objective function that has been used in this paper is demonstrated in equation (52). This robust approach can be obtained by solving equation (52). Where $\mathrm{J}_{1}=\mathrm{d} \omega_{3}, \mathrm{~J}_{2}=$ $\mathrm{d} v_{3}$ and $\gamma=\mathrm{t}_{\text {sim }}$ which $\mathrm{t}_{\text {sim }}, \mathrm{d} \omega_{3}, \mathrm{~d} v_{3}$, and $k$ are the simulation time, angular velocity deviation, terminal voltage deviation, and weighting parameter (in this case $\beta_{1}=7$ and $\beta_{2}=$ 3) respectively. Therefore, the design problem can be formulated as the following optimization problem:

$$
\begin{gathered}
\text { Min } F=\int_{0}^{\gamma} \gamma \times\left(\beta_{1} J_{1}+\beta_{2} J_{2}\right) d \gamma \\
\text { s.t. } 0.15=T_{1}^{\min }<T_{1}=T_{3}<T_{1}^{\max }=15 \\
0.05=T_{2}^{\min }<T_{2}=T_{4}<T_{2}^{\max }=12 \\
3=k^{\text {min }}<k<k^{\max }=120
\end{gathered}
$$

\section{Simulation and Results}

\subsection{Case Study 1}

First, a three-machine test power system (the IEEE 9-bus standard system, Figure 6) is used in this study for comprehending how the proposed method performs in details [25].

\subsection{Results and discussions of Case 1}

Numbers of scenarios created for perturbation are listed in Table 1. Results of controllability gramians computed for each scenario in case 1 are demonstrated in Figure 7. From Figure 7, it can be seen that the choice of the generator G1 leads to the maximum value of the trace of $W c\left(E_{i}\right)$ for all scenarios and therefore, in general, it means energy illustrates the gramians calculated after placing PSS in the IEEE 9-bus system. In particular for gramians, results show a significant increase in value of the trace, which means that the energy needed to drive controllable state variables, has decreased further for generator 1 and thus controller placement is justified. Thus, the controllable state variables are less if we place controller at generator G1. Figure 8 illustrates the gramian after placing and tuning the PSS at G1 in the case1. It can be seen that the average values of PI on the generator got lower. Even though the generator models are equivalent in nature, this gives the criteria for PSS placement. Once the placement group is identified, then individual generator for PSS placement can be extracted. Further tuning of PSS and 
subsequent placement of PSS on other generators is continued until a full rank gramian is reached.

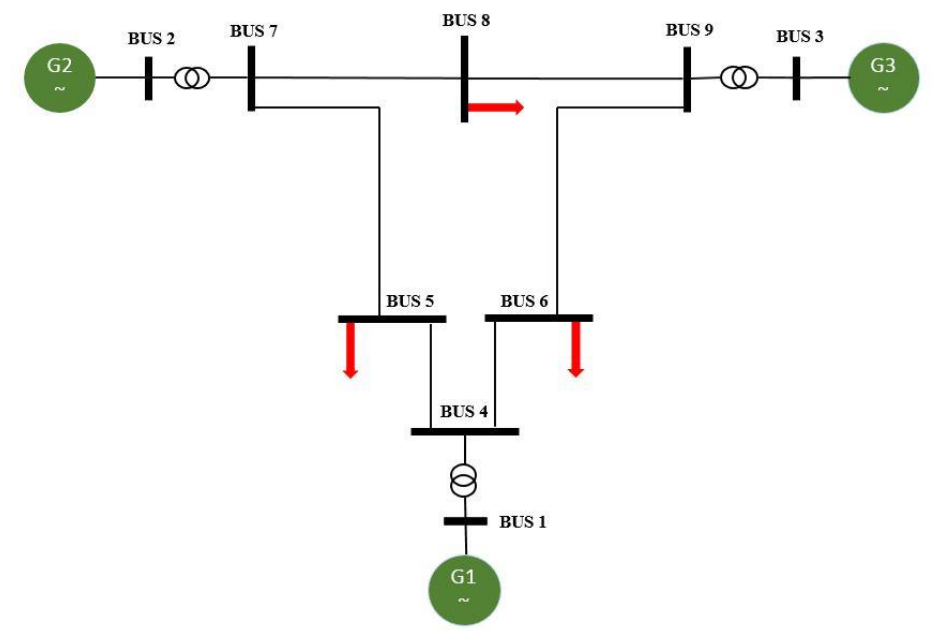

Figure 6. IEEE 9-Bus Test System (Case1)

Table 1. Scenarios Crated for 9-Bus System and 39-Bus System

\begin{tabular}{|c|c|c|}
\hline \multirow{2}{*}{ Scenarios } & \multicolumn{2}{|c|}{ Description } \\
\cline { 2 - 3 } & 9-bus & 39-bus \\
\hline 1 & Without any disturbance & Without any disturbance \\
\hline 2 & Trip in line 4-5 & Trip in line 4-14 \\
\hline 3 & Trip in line8-9 & Increase all Loads by 5\% \\
\hline 4 & Increase all Loads by 10\% & $\begin{array}{c}\text { Increase all Loads by 5\% } \\
\text { and Trip in line 4-14 }\end{array}$ \\
\hline 5 & $\begin{array}{c}\text { Increase all Loads by 10\% } \\
\text { and Trip in line 4-5 }\end{array}$ & $\begin{array}{c}\text { Increase all Loads by 20\% } \\
\text { and Trip in line 4-14 }\end{array}$ \\
\hline
\end{tabular}

The dynamic response of the rotor angle deviation, angular velocity deviation and terminal voltage deviation are shown in Figure 9, Figure 10 and Figure11. It is clearly seen that the PSS1 which has been designed by the ESMIB model for all states is improved and enhanced the electromechanical damping characteristics of G1. Also, Figure 12 shows the system dominant eigenvalues for case1 which clear that the oscillations of the system are damped appropriately by implementing proposed approach to PSS allocation and design in G1.

In this case, the PSSs parameter tuning problem is formulated as an optimization problem and COA is used to seek for optimal parameters. An eigenvalue based objective function reflecting the combination of damping factor and damping ratio is optimized for various operating conditions. The obtained results confirm the robustness and superiority of the proposed stabilizer in providing good damping characteristic to system oscillations over a wide range of loading conditions. 


\subsection{Case Study 2}

Secondly, a 10-machine test power system (the IEEE 39-bus standard system, Figure 13) is used for validating the proposed method in a larger power system [27].

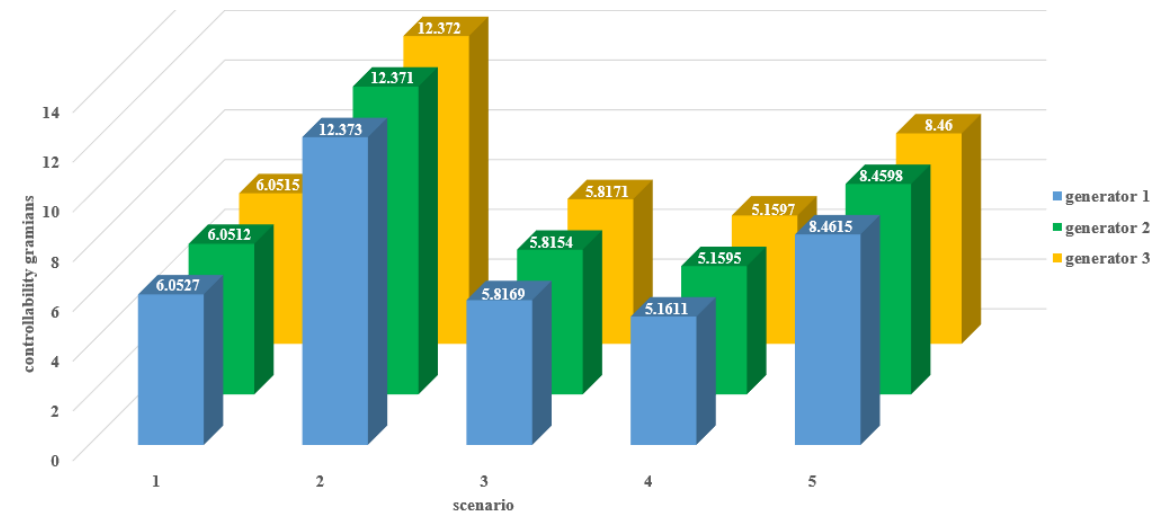

Figure 7. Controllability Gramians Corresponding to Control Inputs for Different Scenarios after PSS Allocation in Case 1

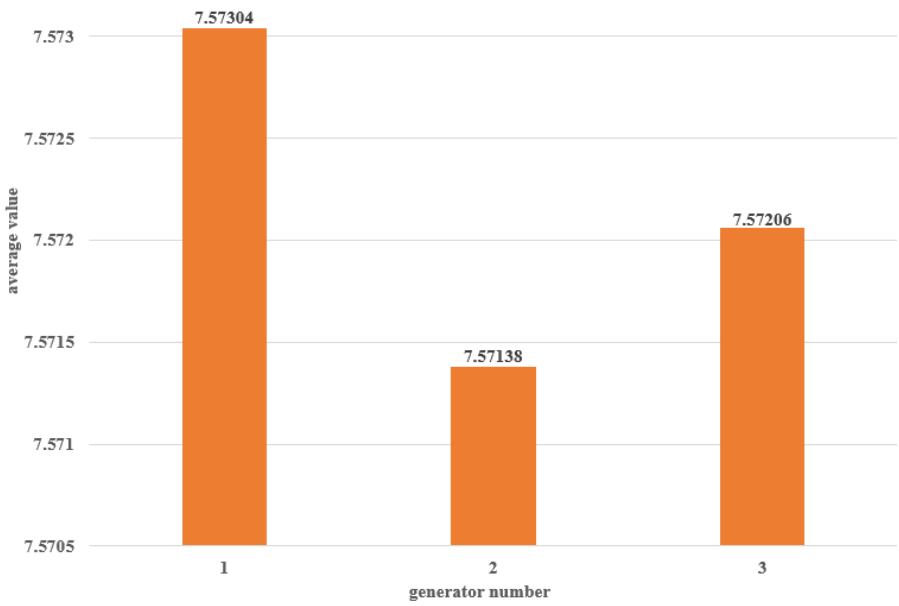

Figure 8. Average Value of for All Machines after PSS Allocation in Case1

Table 2. The Optimized Parameters of the PSS1 in Case1

\begin{tabular}{|c|c|c|}
\hline \hline Parameter & SMIB & ESMIB \\
\hline $\mathrm{T} 1=\mathrm{T} 2$ & 7.732 & 3.895 \\
\hline $\mathrm{T} 3=\mathrm{T} 4$ & 6.431 & 3.274 \\
\hline $\mathrm{k}$ & 68.125 & 88.347 \\
\hline \hline
\end{tabular}




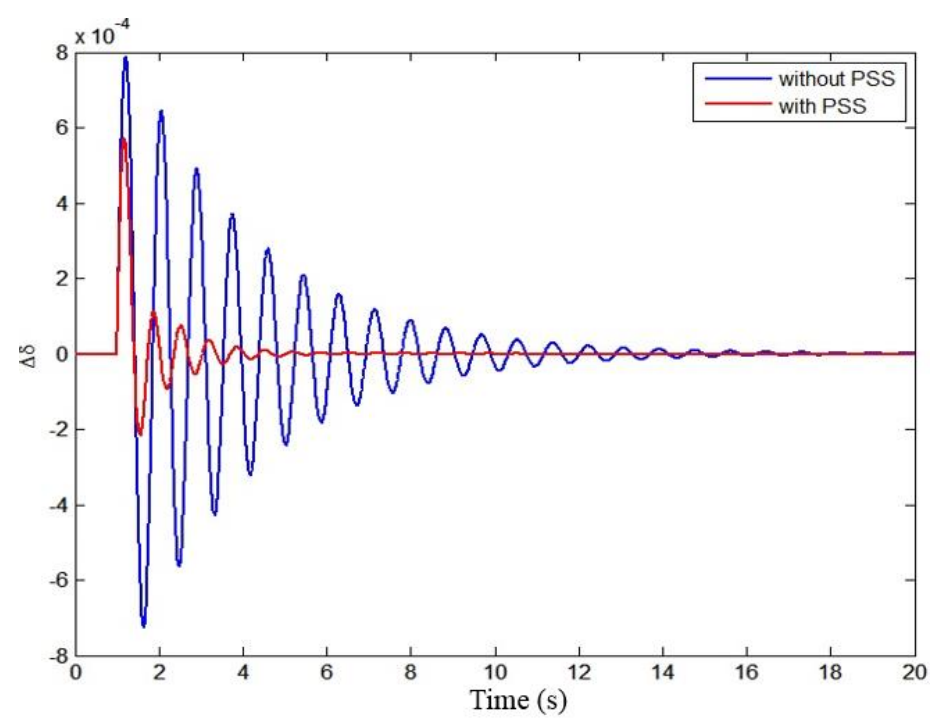

Figure 9. System Response with and without PSS at Stable Operating Points in IEEE 9-bus Test System

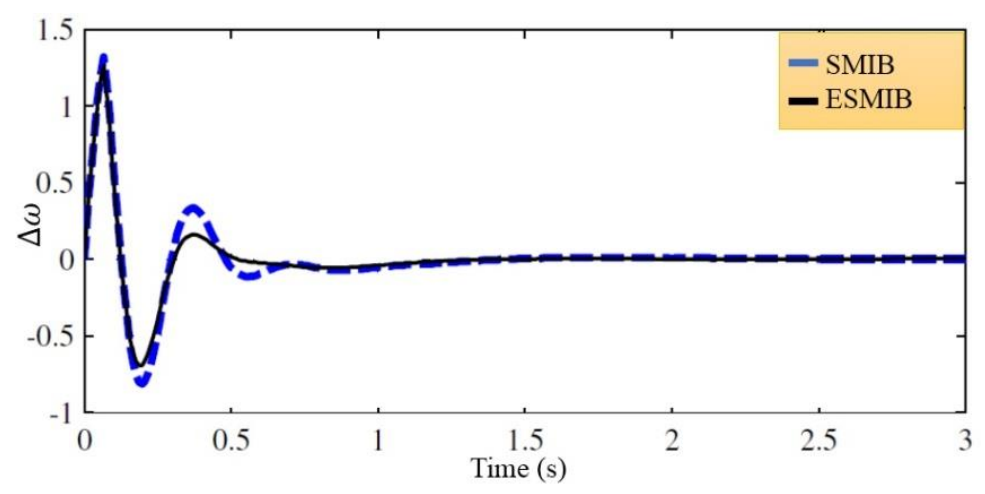

Figure 10. The Dynamic Responses of Angular Velocity Deviation (Pu)

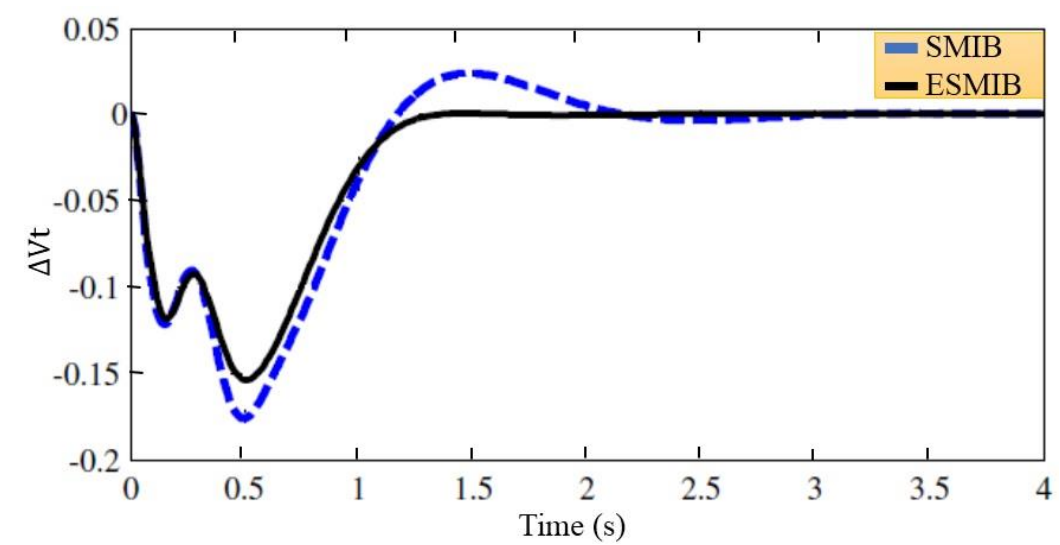

Figure 11. The Dynamic Responses of Terminal Voltage Deviation (Pu) 


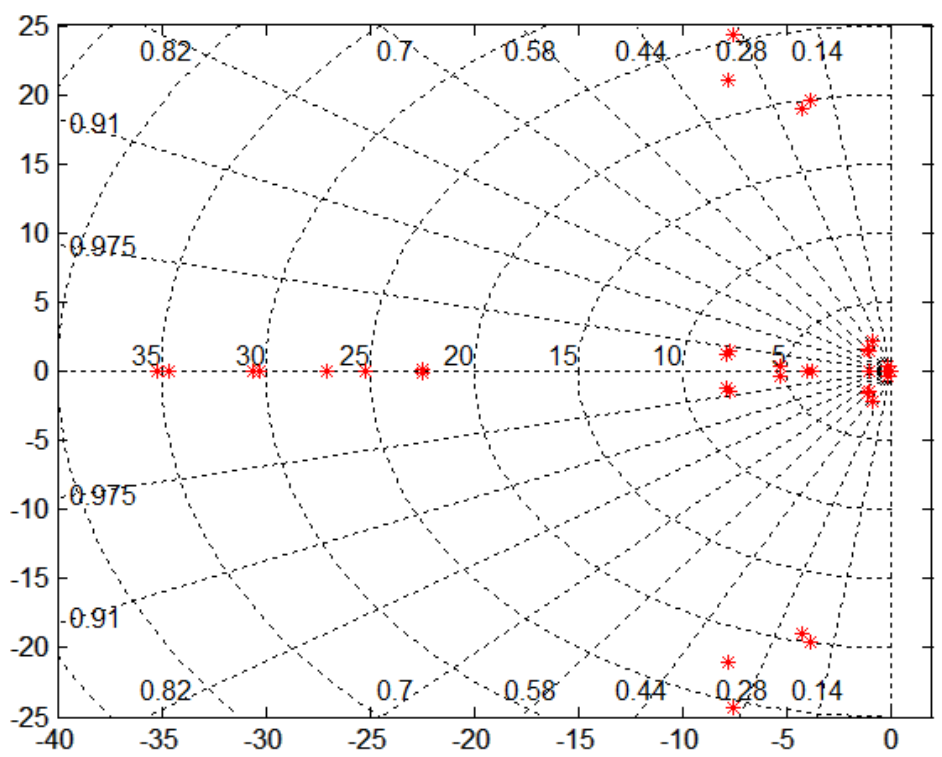

Figure 12. System Dominant Eigenvalues in IEEE 9-Bus Test System

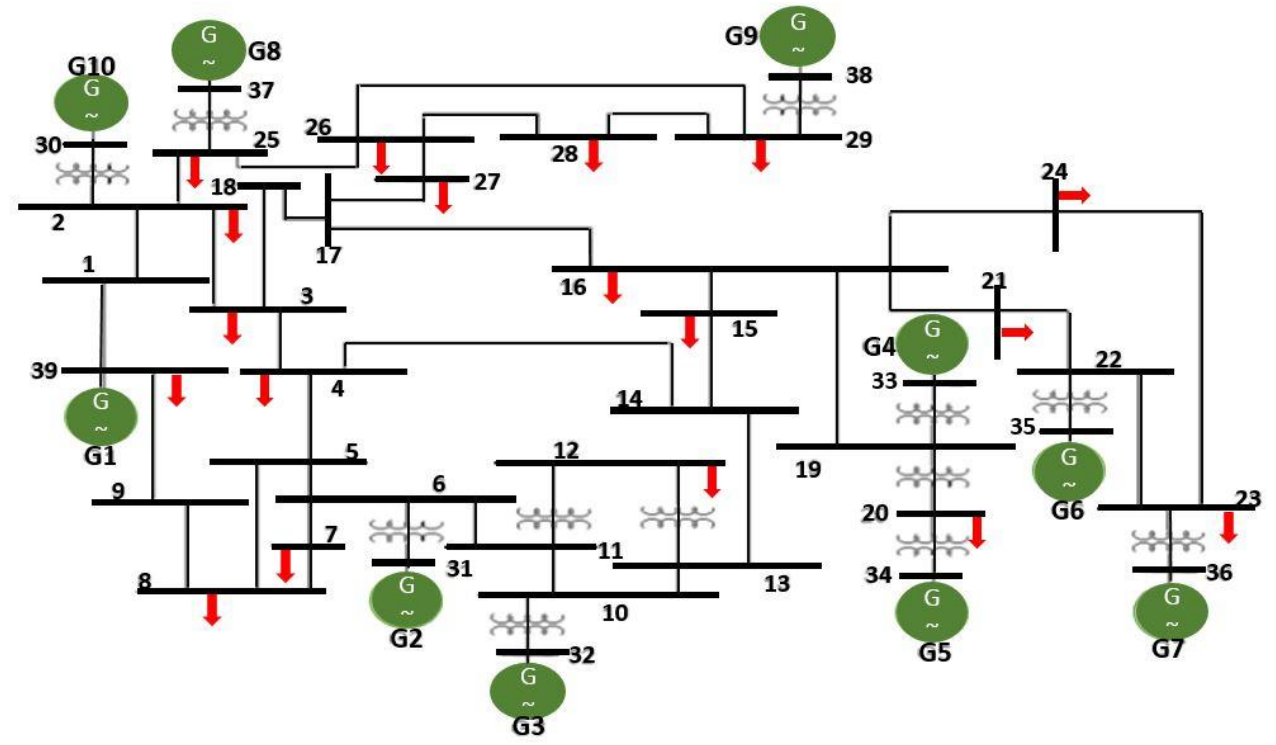

Figure 13. IEEE 39-Bus Test System (Case2)

\subsection{Results and Discussions of Case 2}

For validating the performance of the proposed approach, another nonlinear simulation has been performed. As shown in Figure 13, IEEE 39-bus standard system has been used for this reason. The proposed approach has been utilized for determining the dominant generator in this system (as a result G4 has been chosen for supplying PSS) and the proposed linear model has been used for determining the parameters of the PSS4.Results for gramians depicts that the value of trace of controllability gramians is maximized when we consider G4 as a control input. That means energy required to drive state variable would be minimized if we place controller at G4. Results of controllability gramians computed in case 2 for each scenario are demonstrated in Figure 14. Again, the indexes are computed after placing PSS at G4. 

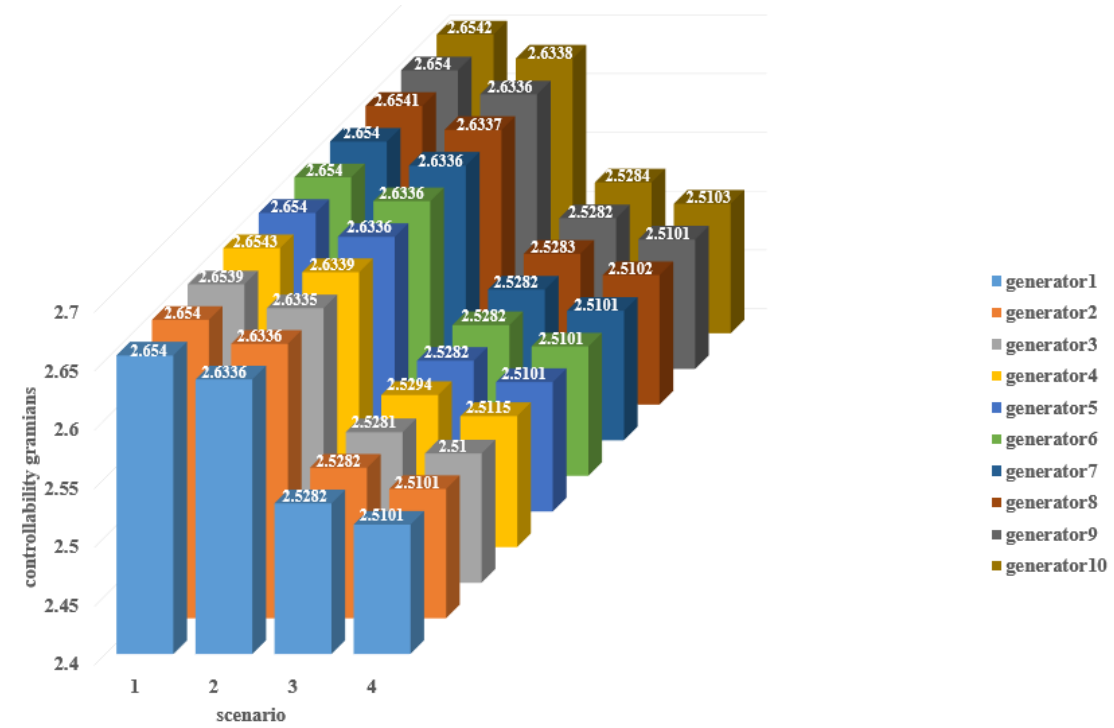

Figure 14. Controllability Gramians Corresponding to Control Inputs for Different Scenarios after PSS Allocation in Case 2

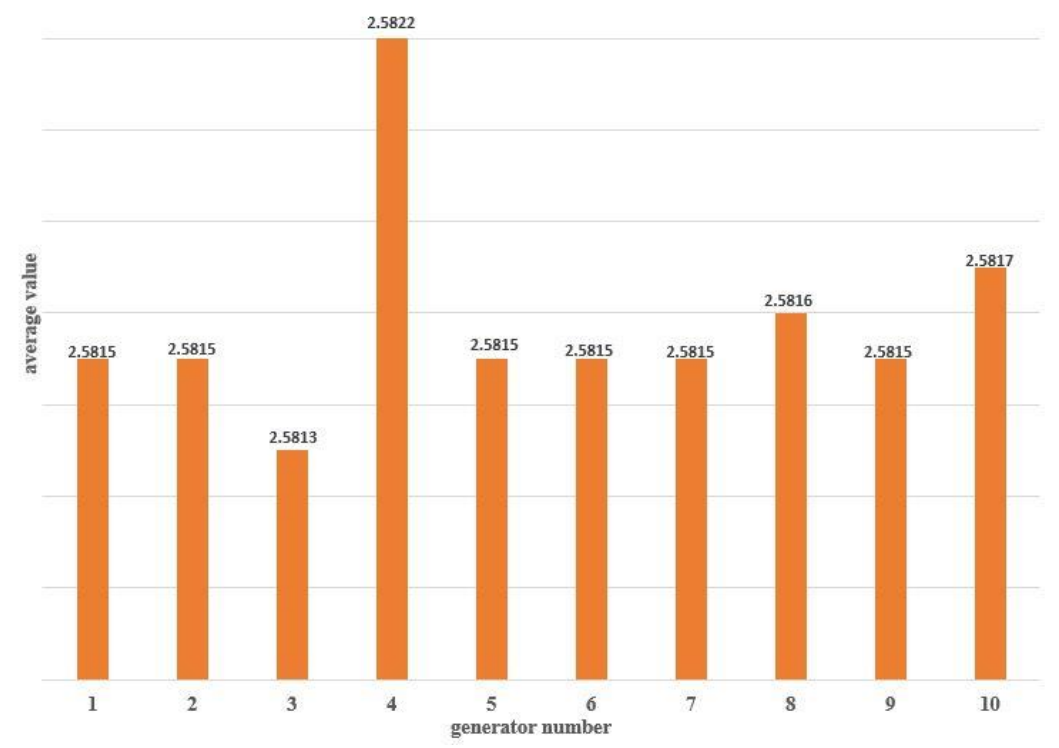

Figure 15. Average Value of for All Machines after PSS Allocation in Case 2

Figure 15 illustrates the gramian after placing and tuning the PSS at G4 in the case 2. It can be seen that the proposed method provides substantial damping to the system. After finding the dominant generator in the multi-machine power system for supplying the PSS via eigenvalue analysis, the PSS has been designed based on the proposed extended linear model of the dominant generator regarding the oscillation modes of power system (local and inter-area modes). Figure 16 clearly depicts that installation and designing PSS based on the proposed approach and linear power system model in the IEEE 39-bus system. It is a very effective way to better damp low-frequency oscillations and leads to enhanced electromechanical damping traits of the system. As a result, it can be concluded that the proposed method can apply to any large power systems. After determining the parameters of the PSS1 by the proposed ESMIB model, in this section for understanding the performance of the ESMIB model, the parameters of the PSS1 are designed by the SMIB model. All conditions are at the same of the ESMIB model. The optimized parameters of case 2 are presented in Table 3. Typically, for different operating conditions, the root 
locus of the system for changing the time delay within the specified limits of the first and second scenarios is shown in Figure 17. According to this Figure, it can be seen that the maximum delay in which the system is stable, is more in the first scenario than the other scenarios. This indicates that in the first scenario, the inter-area mode has more distance from instability point.

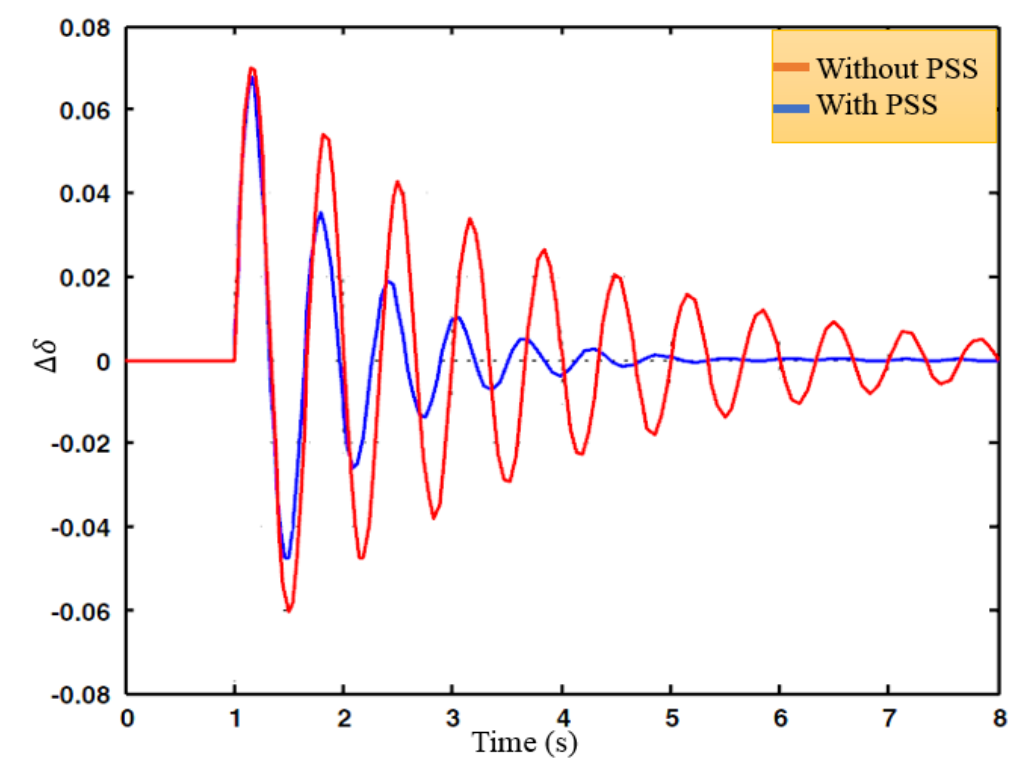

Figure 16. System Response with and without PSS at Stable Operating Points in IEEE 39-Bus Test System

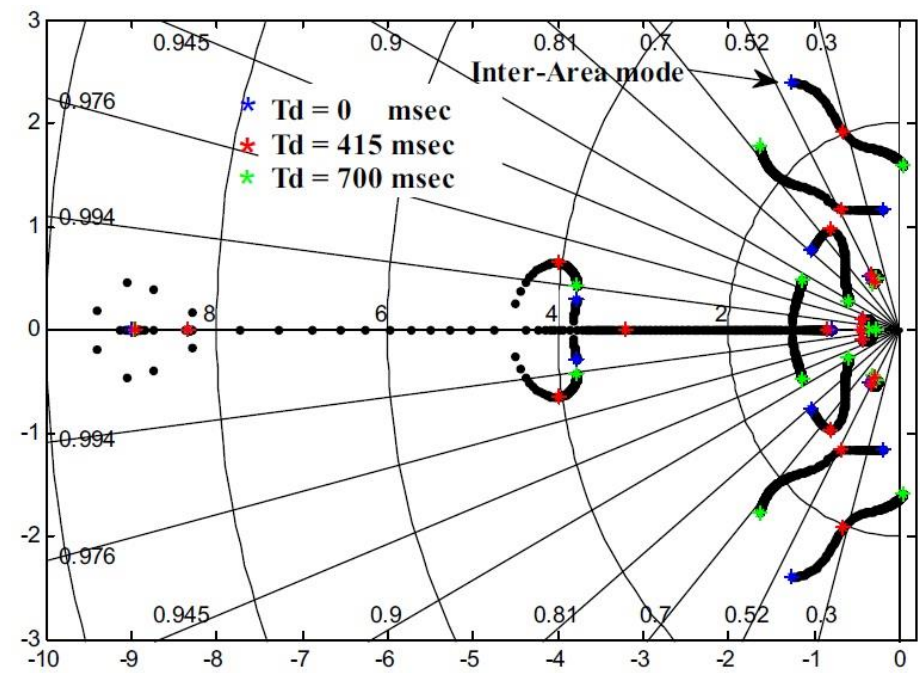

Figure 17. The Root Locus in IEEE 39-Bus Test System with the Time Delay Variations

Table 3. The Optimized Parameters of the PSS4 in Case2

\begin{tabular}{|c|c|c|}
\hline \hline Parameter & SMIB & ESMIB \\
\hline $\mathrm{T} 1=\mathrm{T} 2$ & 9.243 & 4.575 \\
\hline $\mathrm{T} 3=\mathrm{T} 4$ & 8.663 & 4.684 \\
\hline $\mathrm{k}$ & 57.345 & 83.502 \\
\hline
\end{tabular}


The convergence curves of applied COA based optimization algorithm and other conventional optimization algorithms such as Particle Swarm Optimization (PSO) and Genetic Algorithm (GA) have been illustrated in Figure 18. With respect to this figure, it is clear that the COA decreases over iterations faster those other algorithms. As regard to this figure it can be seen that the COA converges quickly compared to PSO and GA. Furthermore, computational time (CPU) of utilized algorithm is compared based on the average CPU time taken to converge the solution so that the average CPU for COA is 20 seconds while the implementation times for PSO and GA are 43 and 58 seconds, respectively. The proposed optimization process is programmed via MATLAB software environment running on an Intel Xeon CPU E5-1620 3.6 GHz laptop with 8 GB RAM. The mentioned CPU time is the average of 20 executions of the computer code.

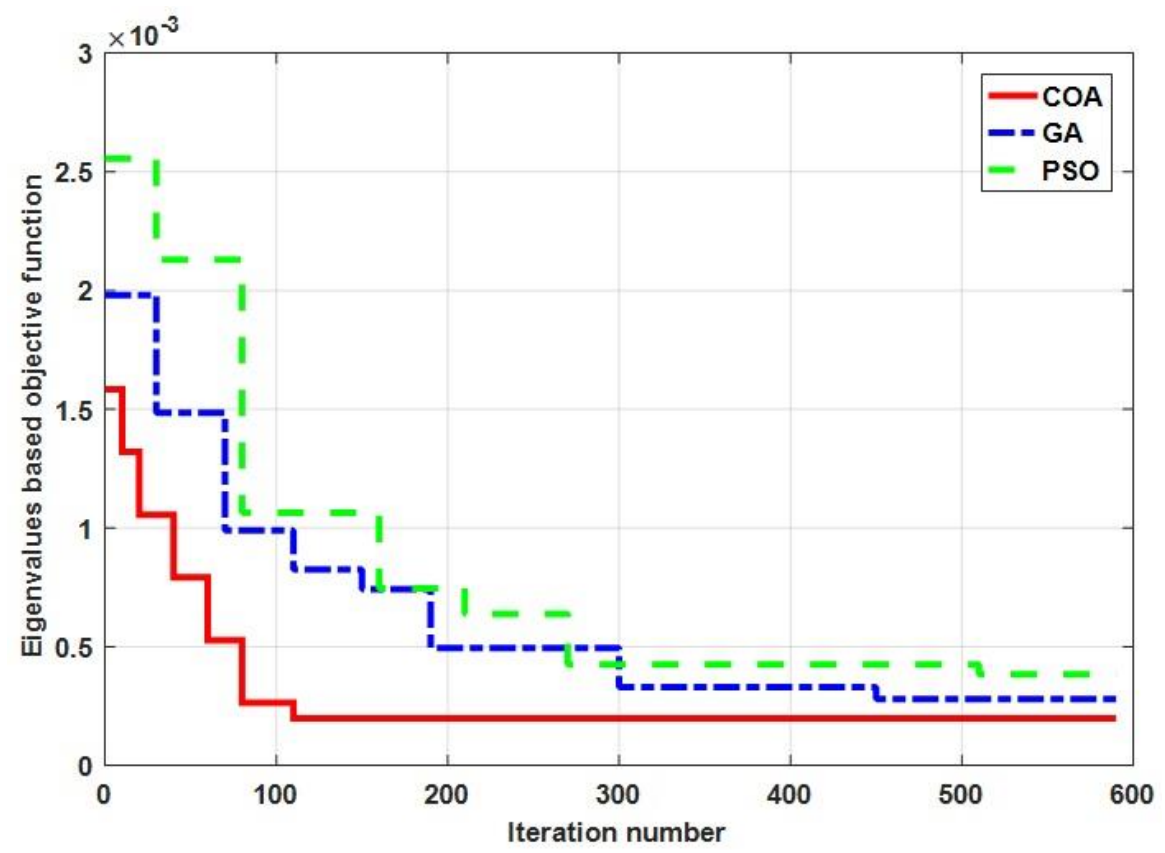

Figure 18. Comparison Between Convergence Curves of Proposed COA and Other Conventional Algorithms

Generally, the privileges and benefits of the proposed model can be concluded as follows:

1. The architecture allows any feedback signal for optimal placement and thus inherently contributes towards optimal location without proper input/output signal.

2. The architecture provides and iterative way to place and tune the PSS on all possible generators till the full controllability of the system is reached.

3. This method provides the participation of the generator with and without PSS and thus provides a way for better controllability and tuning.

4. The proposed model can consider both local and inter-area oscillations of the power network. Designed PSSs based on the proposed extended model improved damping performance of PSSs in comparison with SMIB model.

5. The proposed model provides full rank controllability and thus the PSS placement is the most optimal. 


\section{Conclusions}

This paper proposed a new bi-level approach for optimal robust allocation of PSSs as well as a novel linear model (ESMIB) to design PSSs with regard to the local and interarea modes in order to damp power system oscillations. Allocation and design procedures of PSSs have been applied to the three machines (IEEE 9-bus) and the ten machines (New England test System) test Systems. Based on allocate, the controllability gramian and participation factor method has been introduced to identify dominant generators for installing PSSs. Among the eigenvalues identified, two dominant modes, low-frequency dominant mode have been focused and stabilized. Different disturbances like load deviations and short circuit were considered to evaluate the viability and the robustness of the proposed model. The proposed approach succeeded in determining the dominant generator with the ability to influence these modes by evaluating the participation factors to the low-frequency dominant and stability dominant modes. After finding the dominant generator, the PSS should be designed based on the linear model of this generator. The proposed objective function for PSS tuning is minimized by cuckoo optimization algorithm. The obtained results in both employed systems show that the system composed with the proposed controller can provide an excellent capability in fast damping of power oscillations and improve greatly the dynamic stability of the power system. Furthermore, the nonlinear simulation results illustrated that the designed PSSs based on ESMIB model were capable of guaranteeing the robust performance of the power system for a wide range of operating conditions and different disturbances. Finally, it can be said that the damping of the SMIB power system can be significantly enhanced with ESMIB model and the proposed procedure can be applied to any power systems.

\section{References}

[1] L. Yu-Jen, "Proportional plus derivative output feedback based fuzzy logic power system stabilizer", Int J Electr Power Energy Syst, vol. 44, (2013), pp. 301-317.

[2] H. Shayeghi, S. Jalilizadeh, H. Shayanfar and A. Safari, "Simultaneous coordinated designing of UPFC and PSS output feedback controllers using PSO", Journal of Electrical Engineering, vol. 60, no. 4, (2009), pp. 177-184.

[3] M. Eremia and M. Shahidehpour, "Handbook of electrical power system dynamics:modeling, stability, and control", 1st ed. Wiley-Blackwell, (2013).

[4] S.-M. Baek and J.-W. Park, Hiskens, "Optimal Tuning for Linear and Nonlinear Parameters of Power System Stabilizers in Hybrid System Modeling", IEEE Trans. on Industry Applications, vol. 45, no. 1, (2014), pp. 87-97.

[5] J. Machowski, J. Bialek, J. Bumby.Power system dynamics stability and control. 2nd ed. Wiley; (2010).

[6] S. M Abd Elazim and E. S. Ali, "Optimal Power System Stabilizers design via Cuckoo Search algorithm", Int J Electric Power Energy Syst, vol. 75, no. 3, (2016), pp. 99-107.

[7] R Segal, A Sharma and M. L. Kothari, "A self-tuning power system stabilizer based on artificial neural network", Int J Electric Power Energy Syst, vol. 26, no. 6, (2004), pp. 423430.

[8] A. Dysko, W. E. Leithead and J. Reilly, "Enhanced power system stability by coordinated PSS design", IEEE Trans Power Syst, vol. 25, (2010), pp. 413-422.

[9] N. Johansson, L. Ngquist and H. Nee, "An adaptive controller for power system stability improvement and power flow control by means of a thyristor switched series capacitor (TSSC)", IEEE Trans Power Syst, vol. 25, (2010), pp. 381-391.

[10] A Pal, S. Thorp James, S. Veda Santosh and V. A. Centeno, "Applying a robust control technique to damp low frequency oscillations in the WECC", Int Electr Power Energy Syst, vol. 44, (2013), pp. 638-645.

[11] A. Khodabakhshian, R. Hemmati, Robust decentralized multi-machine power system stabilizer design using quantitative feedback theory. Electric Power Energy Syst, 41 (2012):112-119.

[12] A. Safari, H. A. Shayanfar, A. Kazemi, Robust PWMSC Damping Controller Tuning on the Augmented Lagrangian PSO Algorithm. IEEE Trans Power Syst, 5(1) (2013):1-9. 
[13] SM Radaideh,IM Nejdawi, MH Mushtaha, Design of power system stabilizers using two level fuzzy and adaptive neuro-fuzzy inference systems. Electric Power Energy Syst, 35 (2012):4756.

[14] N. Rezaei, M. Kalantar, H. A Shayanfar, Y. Alipouri, A. Safari, Optimal IPFC signal selection and damping controller design using a novel current injection model in a multimachine power system. Int J Electric Power Energy Syst, 44 (1) (2013):461-470.

[15] LH Hassan, M Moghavvemi, H. Almurib, K. Muttaqi, H. Du. Damping of lowfrequency oscillations and improving power system stability via auto-tuned PI stabilizer using TakagiSugeno fuzzy logic. Electric Power Energy Syst, 58 (2012):72-85.

[16] M. Jing, W. Tong, Y. Weiyang, W. Zengping. Design of wide-area robust damping controller based on the non-convex stable region for interarea oscillations. Int J Electr Power Energy Syst, 55 (2014):473-480.

[17] AY Abd-Elaziz, ES Ali. Cuckoo search algorithm based load frequency controller design for nonlinear interconnected power system. Int J Electric Power Energy Syst, 73 (2015):632-643.

[18] A Safari, H Shayeghi, H.A Shayanfar. Coordinated control of pulse width modulation based AC link series compensator and power system stabilizers. Int J Electric Power Energy Syst,83 (2) (2016):117-123.

[19] S. M. Abd-Elazim, ES. Ali, A hybrid particle swarm optimization and bacterial foraging for optimal power system stabilizers design. Int J Electr Power Energy Syst, 46(1) (2013):334341.

[20] R. H. Yeu, "Small Signal Analysis of Power Systems: Eigenvalue Tracking Method and Eigenvalue Estimation contingency screening for DSA, in ECE Dept.", Unviersity of Illinois at Urbana-Champaign: Urbana, IL, 2010.

[21] E. S. Ali and S. M. Abd-Elazim, "Power system stability enhancement via bacteria foraging optimization algorithm”, Int Arab J Sci Eng, vol. 38, no. 3, (2013), pp. 599-611.

[22] E. S. Ali and S. M. Abd-Elazim, "Coordinated design of PSSs and TCSC via bacterial swarm optimization algorithm in a multimachine power system", Int J Electric Power Energy Syst vol. 36, no. 1, (2012), pp. 84-92.

[23] A. Safari and N. Rezaei, "Towards an extended power system stability: An optimized GCSCbased inter-area damping controller proposal", Int J Electric Power Energy Syst, vol. 56, no. 2, (2014), pp. 316-24.

[24] E. S. Ali and S. M. Abd-Elazim, "Coordinated design of PSSs and SVC via bacteria foraging optimization algorithm in a multimachine power system”, Int J Electr Power Energy Syst, vol. 41, no. 1, (2014), pp. 44-53.

[25] H. Shayeghi, H. A. Shayanfar, A. Safari and R. Aghmasheh, "A robust PSSs design using PSO in a multimachine environment”, Int J Energy Convers Manage, vol. 51, no. 4, (2010), pp. 696-702.

[26] R. Rajabioun, “Cuckoo Optimization Algorithm”, Applied Soft Computing, vol. 11, (2011), pp. 5508-5518.

[27] M. J. Alden and X. Wang, "Robust Hळ control of time delayed power systems", Syst Sci Contr Eng: Open Access J, vol. 3, no. 1, (2015), pp. 253-261.

\section{Authors}

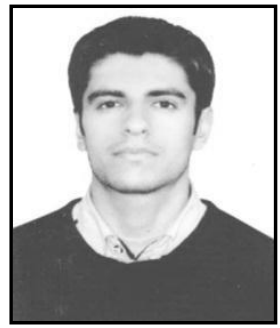

Farhad Samadi Gazijahani, He received the M.Sc. Degree in Electric Power Engineering from Azarbaijan Shahid Madani University, Tabriz, Iran, in 2017. His current research interests include microgrids planning and operation, power system dynamic and control, electricity markets and optimization techniques in power systems. 


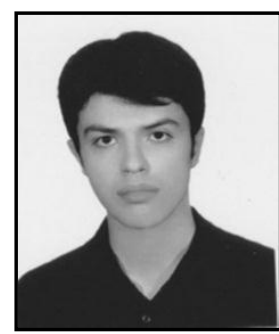

Ata Ajoul Abadi, He received the M.Sc. Degree in Electric Power Engineering from Azarbaijan Shahid Madani University, Tabriz, Iran, in 2017. His current research interests include electrical distribution network and power system dynamic and control.

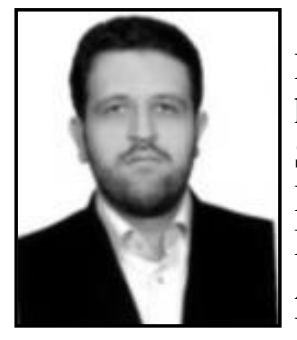

Amin Safari, Hereceived the B.Sc. and M.Sc. degrees in Electrical Engineering in 2007 and 2009, respectively. He received his Ph.D. degree in Power Electrical Engineering, Iran University of Science and Technology, Tehran, Iran. Currently, he is an Assistant Professor in Department of Electrical Engineering, Azarbaijan Shahid Madani University, Tabriz, Iran. His areas of interest in research are Application of artificial intelligence to power system control design, FACTS device and fuzzy sets and systems. He has published more than 100 papers in international journals and conference proceedings. 
International Journal of Control and Automation Vol.10, No.9 (2017) 